\section{Versorgungsbedarf von COPD- und ILD-Patienten}

Spruit MA et al. Pulmonary rehabilitation, physical activity, respiratory failure and palliative care. Thorax 2019; 74: 693 - 699

Ein internationales Autorenteam stellte anhand von Publikationen v.a. aus den Jahren ab 2016 einen Überblick zu den aktuell vorgeschlagenen und untersuchten Maßnahmen zusammen, mit denen die Situation von Patienten mit COPD oder interstitieller Lungenkrankheit (ILD) verbessert werden soll.

\section{Pulmonale Rehabilitation und Trainingstherapie}

Nur 22,6\% der britischen COPD-Patienten werden vor Beginn einer pulmonalen Rehabilitation (PR) mit dem 6-MinutenGehtest (6MGT) untersucht, obwohl davon die angestrebte Trainingsintensität abhängt. Der Sit-to-Stand-Test zeigte ein starke Korrelation zum 6MGT und war prädikativ für die Langzeitergebnisse einschließlich Mortalität. Häufig werden bei COPD-Patienten, die an einer PR teilnehmen, Schmerzen, Fatigue und kognitive Einschränkungen nicht beachtet. Auch bei kognitiven Einschränkungen profitieren die Patienten von der PR. Sozioökonomische Nachteile wirken sich nicht negativ auf eine PR aus. Dagegen hat soziale Unterstützung einen sehr großen Einfluss: Patienten mit einem Ehepartner oder einer Betreuungsperson nehmen mit 11-fach höherer Wahrscheinlichkeit an einer PR teil. Ein in die PR integriertes Armtraining erhöhte Kraft und Ausdauer der oberen Extremitäten und erleichterte Alltagsaktivitäten. Besonders Patienten mit Gleichgewichtsproblemen und schwachen Beinen profitierten von einem GanzkörperVibrationstraining.

\section{Körperliche Aktivität und Trainingstherapie}

Verringerte körperliche Aktivität verschlechtert die Schlafqualität. Darüber hinaus erhöht eine überwiegend sitzende Lebensweise (Energieverbrauch $<1,5$ metabolisches Äquivalent über > 8,5 Stunden/Tag) die 5-Jahres-Sterblichkeit von COPD-Patienten. Während im Rahmen einer PR ein rein Schrittzähler-gestützter Ansatz erfolglos blieb, ließ sich mit einem halb-automatisierten Tele-Coaching inklusive einer App und eines Schrittzählers die Intensität körperlicher Aktivität steigern. Durch eine Sauerstoffsupplementierung (10l/min) konnte die Trainingsleistung bei 29 Patienten mit nicht hypoxischer COPD im Vergleich zu Raumluftzufuhr verdoppelt werden. Bei hypoxischer COPD führte die Sauerstoffzufuhr (2l/min) im Vergleich zu Raumluft beim 6MGT zu verbesserter Leistungsfähigkeit und Sauerstoffsättigung und reduzierte gleichzeitig Dyspnoen sowie ungewollte Pausen.

\section{Ernährungsinterventionen}

Da mangelernährte COPD-Patienten länger im Krankenhaus bleiben müssen und höhere Kosten verursachen, könnten die Krankenaufnahmen der geeignete Zeitpunkt sein, um Ernährungsmaßnahmen zu starten. Neben verringerter Muskelmasse wiesen stationär aufgenommene COPD-Patienten auch sehr häufig Mängel an Vitamin D, B12 und Eisen auf. Eine 3-monatige Ernährungstherapie mit Proteinen, Vitamin D und Omega-3-Fettsäuren verbesserte gegenüber einer isokalorischen Diät den Blutdruck, Lipide und trainingsbedingte Erschöpfung. Es wird die Kombination aus multimodaler Ernährungstherapie und körperlichen Übungen empfohlen.

\section{Nicht invasive Beatmung (NIV) und Atemnot}

Die Langzeit-NIV führte bei COPD-Patienten zu deutlich reduziertem arteriellen Kohlendioxidpartialdruck $\left(\mathrm{PaCO}_{2}\right)$ und reduzierter Mortalität. Dabei wurde die NIV gut toleriert und verbesserte die Lebensqualität. Die bei fortgeschrittener Krankheit auftretende Atemnot kann effektiv mit Opioiden verbessert werden. Kurzzeitig eingesetzt, verbesserten diese Medikamente statistisch signifikant und klinisch relevant die Schwere der Atemnot. Die Kurzzeittherapie mit Opioiden war nicht mit respiratorischen Nebenwirkungen verbunden.

ILD-Patienten tragen eine hohe Krankheitslast, besonders durch Atemnot. Die Beachtung der Atemnotbehandlung als Zusatz zur ILD-gerichteten Therapie nimmt zu, u.a. indem die chronische Atemnot als neues klinisches Syndrom betrachtet wurde. Atemnot verschlechtert weitreichend die Lebensqualität und sollte ganzheitlich beurteilt und behandelt werden.

FAZIT

Die pulmonale Rehabilitation hilft zwar nicht allen Patienten, ihr Nutzen wurde aber in aktuellen Metaanalysen und Leitlinien insgesamt bestätigt. Die PR könnte bei Patienten mit fortgeschrittenen chronischen Atemwegserkrankungen u. a. ergänzt werden durch die High-Flow-Sauerstofftherapie oder Biologika bei schwerer COPD.

Matthias Manych, Berlin 